\title{
Data Persistence on Curriculum Management System based on MyBatis
}

\author{
Li Ma \\ School of Education and Sports, Bohai University, Jinzhou, 121013, China \\ 88228471@qq.com
}

Keywords: MyBatis; curriculum management system; data persistence; functional architecture; data store structure

\begin{abstract}
Course of information management and development of curriculum management system is to reduce the educational task complexity and the effective ways to improve the efficiency of the educational administration work. Data persistence is an important work of software development, this article is based on MyBatis technology research and provide technical support for the system development. First of all, research by the interface layer, data processing layer and foundation support layer of MyBatis function architecture; Then, based on the Sybase database management system, curriculum information table, for example, for data storage logic structure design; Finally, to design the persistence code, including total configuration files, object mapping files and entity class object. The actual application, attention should be paid to give full play to the advantage of the MyBatis, improve the overall performance of the system and the development efficiency.
\end{abstract}

\section{Introduction}

Course management is an important educational administration work, involves the teaching plans, course arrangement and performance management aspects of work, is one of the most complex work of educational administration system. Was carried out on the course information management, development of curriculum management system, is to conduct standardized management, to reduce the educational task complexity and the effective ways to improve the efficiency of the educational administration work.

Data persistence is a process to convert data in memory model for storage model and converts the storage model in memory, to achieve data in memory and the exchange of data in the database, is an important part of the software system development. Java development the persistent technology is the most commonly used Hibernate and iBatis. IBatis is an Apache open source project, in 2010 by the "Apache software foundation" migrated to Google code ", and changed its name to "MyBatis" [1]. MyBatis is to support the common SQL queries, stored procedures and advanced mapping of excellent persistence layer framework; Eliminates almost all JDBC code, parameter of manual setup and result set retrieval; Using a simple XML or annotations for configuration and the original mapping, the interface and Java POJOs mapping database records. The programmer can follow one's indiscriminately use object to manipulate the database programming thinking, improve software development efficiency and maintainability. In this paper, based on the study of MyBatis, provide technical support to the development of the software.

\section{Functional Architecture on MyBatis}

Mybatis function architecture is divided into three layers, namely, interface layer, data processing layer and foundation support layer, as shown in Fig.1, described the following [2, 3] :

(1) The Interface layer: provide for the use of external Interface API, developers through the API to manipulate the database. The interface layer receives the call request is called data processing layer to complete specific data processing. SqlSessionFactory and SqlSession is the core of the MyBatis interface layer, SqlSessionFactory object by the SqlSessionFactoryBuilder is created, the main function is to create the SqlSession objects; SqlSession object's main function is to complete a database access and mapping as a result, because is not thread safe, so the SqlSession objects need to limit the scope of the method. 
(2) The data processing layer: It is responsible for the specific SQL search, parse SQL, SQL execution and the execution result map processing, and the main purpose is according to the call request to complete a database operation., MyBatis.XML analyses SqlMapConfig.xml and SqlMap.xml Two configuration files when application startup. The SqlMapConfig.xml to complete the analysis in the XMLConfigBuilder class; SqlMapConfig.xml complete parsing in the XMLConfigBuilder class, the involved PreparedMapping, ResultMapping, LanguageDriver, Discriminator, caching, and automatic mapping and so on a series of object structure. Executor is the core in MyBatis with the full process of database operations, need MappedStatement, parameter and result Handler instance objects. Transaction commit/rollback, entrusted to the Transaction object to complete; Cache, create CacheKey ()/is Cached (); Lazy loading, defer load (); Close, close (), mainly is the transaction rollback/closed.

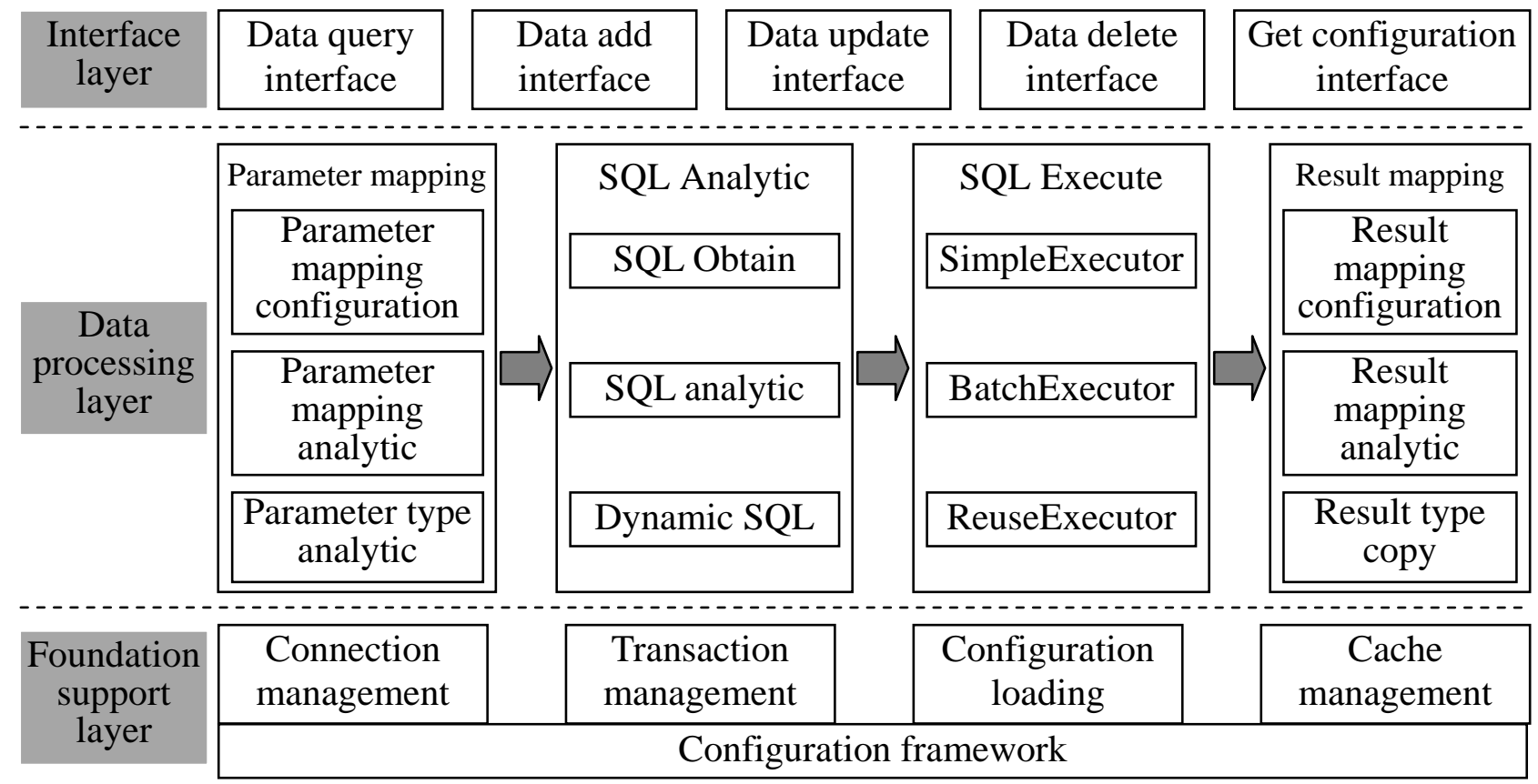

Fig. 1. Functional architecture on MyBatis

(3) The Foundation support layer: It is responsible for the most basic functions, including the connection management, transaction management, configuration, load and cache handling, the Shared component is extracted as the most basic components, provide the Foundation of support for the data processing layer. MyBatis provides a simple data source/connection pool under the org. apache. ibatis.datasourse, implementation class is PooledDataSource, contains the maximum number of connections, the largest number of idle connections, and connection is not enough to take out the longest time waiting time, etc.; MyBatis for transaction processing is relatively simple, TransactionIsolationLevel defines several isolation level, doesn't support nested transaction, application development, entrust the Spring to handle affairs; MyBatis includes two kinds of cache. Session and the Statement is scope level, default is Session, BaseExecutor according to MappedStatement Id, SQL, the value and CacheKey rowBound to construction, and maintenance by using the localCache BaseExccutor cache. Global level 2 cache could be come true through CacheExecutor, and entrust TransactionalCacheManager save or access to the cache.

\section{Logical Structure Design on Data Store}

Logical Structure Design on Data Store associated with the database management system of concrete structure, and choosing the Sybase as course management system. Sybase database is launched in high-performance products of Sybase company, and it is based on client/server architecture, the application is run on the multiple machines. Advantage is to support sharing resources and balance load between multiple devices, allowing for multiple host environment, make full use of the enterprise has some various systems. 
Sybase database is mainly composed of three parts: one is that for database management and maintenance of "Sybase SQL Server", is a programmable database management system, is the core of the Sybase product software, to play the data management, high-speed buffer management, transaction management role; Second, support the establishment of the database application system and the development of "Sybase SQL Toolset, ISQL interact with SQL Server is a SQL syntax analyzer, DWB is convenient and practical database management tools, APT is mainly engaged in the development of the practical application environment; Three is that other manufacturers under heterogeneous environment application software with any type of data connection "the Sybase Open Client/Open Server".

The data storage structure design of curriculum management system includes many tables, and this paper illustrates data persistence approach by using only "CourseComprehensiveInformationTable", logical structure as shown in Table $1(4,5)$.

Table 1. Database logical structure on CourseComprehensiveInformationTable

\begin{tabular}{c|l|c|c|c|l|c|c}
\hline No & FieldName & DataType & Width & No & FieldName & DataType & Width \\
\hline 1 & CourseCode & Char & 16 & 15 & CreditHours & Decimal & 4 \\
\hline 2 & CourseName & Varchar & 50 & 16 & TotalPeriod & Tinyint & 1 \\
\hline 3 & CourseTypeName & Varchar & 50 & 17 & LecturePeriod & Tinyint & 1 \\
\hline 4 & CourseTypeCode & Char & 4 & 18 & ExperimentPeriod & Tinyint & 1 \\
\hline 5 & TeachingTypeName & Varchar & 50 & 19 & StartTeachingSemester & Tinyint & 1 \\
\hline 6 & TeachingTypeCode & Char & 2 & 20 & HoursPerWeek & Tinyint & 1 \\
\hline 7 & ExaminationModeName & Varchar & 50 & 21 & TeachingWeeks & Tinyint & 1 \\
\hline 8 & ExaminationModeCode & Char & 1 & 22 & EnglishTeachingFlag & Tinyint & 1 \\
\hline 9 & TeachingCollegeName & Varchar & 50 & 23 & MultimediaTeaching & Tinyint & 1 \\
\hline 10 & TeachingCollegeCode & Char & 3 & 24 & FlippedClassroomFlag & Tinyint & 1 \\
\hline 11 & TrainingTypeName & Varchar & 50 & 25 & TeachingMaterialName & Varchar & 50 \\
\hline 12 & TrainingTypeCode & Char & 1 & 26 & PublishingCompany & Varchar & 50 \\
\hline 13 & ProfessionalName & Varchar & 50 & 27 & BookNumber & Varchar & 20 \\
\hline 14 & ProfessionalCode & Char & 3 & 28 & CourseDescription & Text & \\
\hline
\end{tabular}

\section{Code Design on Data Persistence}

MyBatis persistence design is mainly the following three [6, 7] configuration file:

(1) Total MyBatis configuration file: the configuration.xml. The configuration file defines the database connection information and global configuration information. Using the configuration file has powerful function, convenient operation, good format check mechanism, automatic file loading, etc. Configuration.xml file code is as follows:

\begin{tabular}{|c|c|}
\hline $\begin{array}{l}\text { es> } \\
\text { as } \\
\text { User" } 1> \\
\text { es> } \\
\text { ents default="development"> }\end{array}$ & $\begin{array}{l}\text { aracterEncoding=UTF-8" /> } \\
\text { ame="username" value="root" /> } \\
\text { ame="password" value="" /> } \\
\text { urce> } \\
\text { ent }> \\
\text { ronments> } \\
\text { ers }> \\
\text { mapper resource="domain/Course.xml" } \\
\text { pers }> \\
\text { tion> }\end{array}$ \\
\hline
\end{tabular}


(2) The object mapping file: courseMapper.xml. Entity class mapping file is used to finish to the operation of the database objects, including to perform a variety of SQL statements, stored procedure, the input parameter mapping, mapping, caching mechanisms, and can through several relatively complex configuration make the relationship between objects and lazy loading, realized the data storage logic from the upper logic code. CourseMapper. xml file code is as follows:

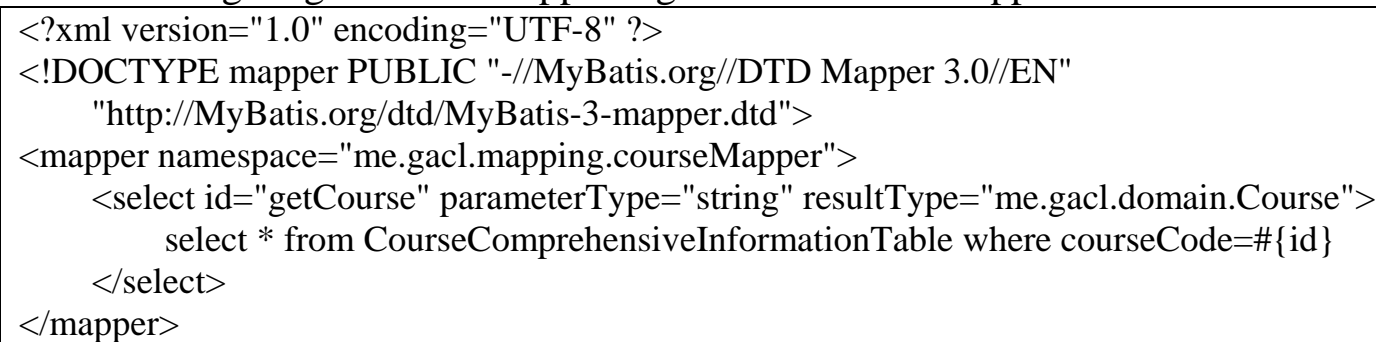

(3) Entity class object file: Course.Java. Entity class object is used to complete the operation of the database, in between the business logic and database resources, improve the independence of the class. Database is required in the operation of each table needs to write the DAO implementation class. The DAO and the business logic layer transfer is an entity object, is a mapping of database tables in the program. For each attribute the get and set methods as access interface, improves the independence of the class. Due to the many properties CourseComprehensiveInformationTable, previously only 10 attributes, for example. Course.Java code is as follows:

\begin{tabular}{|c|c|}
\hline $\begin{array}{l}\text { Iblic class Course }\{ \\
\text { private string courseCode; } \\
\text { private string courseName; } \\
\text { private string courseTypeName; } \\
\text { private string courseTypeCode; } \\
\text { private string teachingTypeName; } \\
\text { private string teachingTypeCode; } \\
\text { private string examinationModeName; } \\
\text { private string examinationModeCode; } \\
\text { private string teachingCollegeName; } \\
\text { private string teachingCollegeCode; } \\
\text { public string getCourseCode() \{ } \\
\text { return courseCode; }\} \\
\text { public void setCourseCode(string courseCode) }\{ \\
\text { this.courseCode = courseCode; }\} \\
\text { public string getCourseName() \{ } \\
\text { return courseName; }\} \\
\text { public void setCourseName(string courseName) }\{ \\
\text { this.courseName = courseName; }\} \\
\text { public string getCourseTypeName() \{ } \\
\text { return courseTypeName; }\} \\
\text { public void setCourseTypeName(string } \\
\text { courseTypeName) }\{ \\
\text { this.courseTypeName = courseTypeName; }\} \\
\text { public string getCourseTypeCode() \{ } \\
\text { return courseTypeCode; }\} \\
\text { public void setCourseTypeCode(string } \\
\text { this.courseTypeCode = courseTypeCode; }\} \\
\text { return teachingTypeName; }\}\end{array}$ & $\begin{array}{l}\text { public void setTeachingTypeName(string } \\
\text { teachingTypeName) \{ } \\
\text { this.teachingTypeName = teachingTypeName; \} } \\
\text { public string getTeachingTypeCode() \{ } \\
\text { return teachingTypeCode; }\} \\
\text { public void setTeachingTypeCode(string } \\
\text { teachingTypeCode) \{ } \\
\text { this.teachingTypeCode = teachingTypeCode; }\} \\
\text { public string getExaminationModeName() \{ } \\
\text { return examinationModeName; }\} \\
\text { public void setExaminationModeName(string } \\
\text { examinationModeName) \{ } \\
\text { this.examinationModeName = } \\
\text { examinationModeName; }\} \\
\text { public string getExaminationModeCode() \{ } \\
\text { return examinationModeCode; }\} \\
\text { public void setExaminationModeCode(string } \\
\text { examinationModeCode) \{ } \\
\text { this.examinationModeCode = } \\
\text { examinationModeCode; }\} \\
\text { public string getTeachingCollegeName() \{ } \\
\text { return teachingCollegeName; }\} \\
\text { public void setTeachingCollegeCode(string } \\
\text { teachingCollegeName) \{ } \\
\text { this.teachingCollegeName = } \\
\text { teachingCollegeName; }\} \\
\text { public string getTeachingCollegeCode() \{ } \\
\text { return teachingCollegeCode; }\} \\
\text { public void setTeachingCollegeCode(string } \\
\text { teachingCollegeCode) }\{ \\
\text { this.teachingCollegeCode = } \\
\text { teachingCollegeCode; }\} \text { \} }\end{array}$ \\
\hline
\end{tabular}

\section{Conclusion}

MyBatis has the following advantages: one is that simple, through the document and source code, can be more fully master the design ideas and implementation; Second, practical, and provide data 
mapping function, access to the underlying data encapsulation and DAO framework, it is easier to develop and deploy DAL layer; The third is, flexible, basic can be achieved through SQL, do not use the data access framework to implement all functions; The fourth is, fully functional, through configuration object mapping relationship between the data access layer to solve the problem, such as DAO support, and encapsulates the Ado.NET in the DAO plane, I got and DataMapper; The fifth is, maintainability, by providing DAL layer, business logic and data access logic separation, the system design more clearly, easier maintenance, easier to unit test. SQL improves the maintainability and code separation. This paper research the actual application, should pay attention to and the combination of these advantages, improve the overall performance of the system and the development efficiency.

\section{Acknowledgement}

This work is supported by 2013 annual social science planning fund project of Liaoning province (L13CTQ012): Research on investigation and cultivation for college students' reading tendency of digital resources; 2014 annual education science planning of Liaoning province (JG14DB019): Strategy research on teachers' teaching mode transformation and teaching ability in digital environment.

\section{References}

[1] Y. X. Huang, "Automatic generation technology of database based on Mybatis," Journal of Henan Science and Technology, vol. 39, no. 4, pp. 21-22, 2014.

[2] Y. D. Rong, "Application Research of Mybatis Persistence Layer Framework," Information Security and Technology, vol. 6, no. 12, pp. 86-88, 2015.

[3] bubuko, "MyBatis architecture design and source code analysis series: MyBatis architecture," http://www.bubuko.com/infodetail-549184.html, 2016-5-22.

[4] P. F. Li, "Design and implementation of management system for comprehensive course in university based on.NET," Master's degree of Yanshan University, 2012.

[5] J. Li, "Design and Implementation of A Curriculum Information Management System for Universities and Colleges," Master's degree of Xiamen University, 2014.

[6] Linux community, "MyBatis entry learning tutorial," http://www.linuxidc.com/Linux/2015-02/113771.htm, 2016-5-22.

[7] Y. M. Shi, "Data paging display processing based on Spring MVC and MyBatis," Information Technology and Informatization, vol. 40, no. 7, pp. 203-206, 2015. 